\title{
First observations of entropy vs free energy for lattice based Ising model for spin coarsening in conserved and non-conserved binary mixtures: a phenomenological study of phase transitions in 2D thin films
}

\author{
Satya Pal Singh \\ Department of Applied Sciences, Madan Mohan Malaviya University of Technology, \\ Gorakhpur, 273010, India \\ singh.satyapal@hotmail.com
}

\begin{abstract}
PACS 75.20.En, 75.70.Kw, 81.30.Bx, 75.10.-b, 75.10.Hk, 75.30.Kz, 64.60.De, 05.40.-a DOI 10.17586/2220-8054-2015-6-6-882-895
\end{abstract}

This paper presents the results of Monte Carlo (MC) simulation for paramagnetic, ferromagnetic and anti-ferromagnetic transitions in 2D thin films. The spin coarsening which lowers energy brings order at the cost of lowering entropy in the presence of an external magnetic field, which in turn, may increase the free energy at relatively higher temperatures because of spin mixing. There is a competition between energy of the system and entropy in conserved and non-conserved binary mixtures in the presence of an external magnetic field. The simulation is done on a lattice of size $100 \times 100$ using Metropolis algorithm with periodic boundary conditions. All data are sampled for $20 \mathrm{~K} \mathrm{MC}$ cycles after a regular interval of $100 \mathrm{MC}$ cycles. The paramagnetic case with spin coupling coefficient and the ferromagnetic and anti-ferromagnetic cases with $|\varepsilon(A B)|=0.0 J_{0}, 0.25 J_{0}, 0.50 J_{0}$, $0.75 J_{0} \& 1.0 J_{0}$ (keeping $|\varepsilon(A A)|=|\varepsilon(B B)|=1.0 J_{0}$ ) (Here $J_{0}=1.0$ unit of energy) are studied at temperatures $k T=0.25 J_{0}, 0.50 J_{0}, 0.75 J_{0}, 1.0 J_{0}, 1.25 J_{0}, 1.5 J_{0}, 1.75 J_{0}, \& 2.0 J_{0}$ in presence of varying external magnetic field strengths in order to observe the organizational behavior of spins and the interplay between the free energy and entropy. The induced magnetization and the magnetic susceptibilities are found to be in qualitative agreement with the theory. The paramagnetic to ferromagnetic transition has been observed and explored at high $T$ values. The spin correlation function plotted helps to reveal the spin transport properties of the systems. The spontaneous ferromagnetic transition temperatures for $\varepsilon(A B)=1.0 J_{0}, 0.75 J_{0} \& 0.50 J_{0}$ are observed as $k_{B} T=0.44 J_{0}, 0.39 J_{0}$ $\& 0.33 J_{0}$ (i.e. nearly $96 \%$ magnetizations are observed at these temperatures) respectively at $B=0.0 J_{0} / \mu$ (i.e. absence of any external magnetic field). This is in quantitative agreement with theory. The spin correlation function diverges at these transition temperatures, which can be understood as the theoretical evidence supporting the observation of spontaneous magnetization. The ferromagnetic to paramagnetic transitions are not very sharp but the range of the spin-spin interaction can be said to decay gradually. Even at higher temperature as $k T=2.0 J_{0}$, the opposite spin pair correlation function supports formation of tiny domains with spin transport from one domain to another, whereas for lower temperatures below $k T=1.0 J_{0}$, the presence of majority $+1 / 2$ spins diminish the effect. Tiny domain walls have lower energy surrounded by opposite spins and seem to be energetically preferred. This quasi nature of spin-spin interaction with temperature is also supported by the corresponding ensemble entropy averages.

Keywords: Ising model, spontaneous magnetization, thin films, spin conservation, super para-magnetism.

Received: 11 September 2015

Revised: 24 October 2015 


\section{Introduction}

Magnetism is an old problem of study arising from the existence of naturally magnetic materials. Perhaps, the maritime use of a compass needle stands among the first few widely used applications of magnetic materials. Magnetic materials have drawn new attention in last few decades because of magnetic properties variability at nanoscale and their interplay with the electrical property because of charge of electronics. Thus, spintronics and magneto-electronics have emerged as new branch of science which can open doors for inventions having the potential for novel industrial applications [1-4].

Because of their extensive applications in diverse fields of electronics, optics, space science, aircrafts, defense and many other industries, thin films have led to a rise in phenomenal research. Molecular Simulation with thin films using lattice model and metropolis algorithm can give new insights into small scale problems with reduced dimensions. In order to understand the underlying physical process on the basis of fundamental principles of physics one can efficiently use computer models and obtain similar results similar to those determined experimentally. The simulation methods can also help to predict the molecular behavior at small scales which can be tested through well designed experiments. In studying phase transitions and sampling the data for physical observables, the concepts of advance statistical mechanics [5,6] and thermodynamics [7] have been found to be very useful. Advanced generations of computers have made it possible to sample data with greater and greater accuracy. This paper deals with the thermodynamic and statistical study of coarse graining process of electron spins $\pm 1 / 2$ in two dimensional thin films with conserved $(A: B:: 50: 50)$ and non-conserved spins (i.e. spin flipflop ensures minimization of free energy). The cases studied are paramagnetic, ferromagnetic $\&$ anti-ferromagnetic.

\section{Methodology}

Monte Carlo (MC) simulations have been used successfully to study a variety of problems ranging from wetting [8-11], dewetting and surface-directed phase separation [11, 12], magnetic properties of thin films etc. [13-16]. In this paper, the spin-spin interactions are calculated in the presence of external magnetic field to sample the ensemble average of internal energy and entropy. The configurational interaction energy is given by eq. (1) [17]:

$$
H\left\{S_{i}\right\}=-\sum_{<i j>} \epsilon_{i j} S_{i} S_{j}-B \sum_{i} S_{i}
$$

The initial configuration is generated by placing $\pm 1 / 2$ i.e. spin up \& spin down on a $100 \times 100$ lattice in a random fashion so that the two types of spin populate in the ratio $1: 1$. The spin flip-flop move is accepted if $\exp \left(-\Delta E / k_{B}\right) T \geq p$ in case of non-conserved case. Here, $p$ is a random number chosen between $0-1$. The initial configuration is kept at high temperature $k_{B} T=10.0 J_{0}$ for first $1 \mathrm{~K} \mathrm{MC} \mathrm{Cycles} \mathrm{and} \mathrm{then} \mathrm{is} \mathrm{equilibrated} \mathrm{at} \mathrm{the} \mathrm{given} \mathrm{temperature} \mathrm{for}$ $10 \mathrm{~K} \mathrm{MC} \mathrm{Cycles.} \mathrm{The} \mathrm{sampling} \mathrm{for} \mathrm{the} \mathrm{thermodynamic} \mathrm{observables} \mathrm{as} \mathrm{the} \mathrm{internal} \mathrm{energy}$ and entropy, magnetization and spin correlation starts after $10 \mathrm{~K} \mathrm{MC} \mathrm{Cycles.} \mathrm{The} \mathrm{sampling}$ is done for next $10 \mathrm{~K} \mathrm{MC} \mathrm{Cycles} \mathrm{after} \mathrm{a} \mathrm{regular} \mathrm{interval} \mathrm{of} 100 \mathrm{MC}$ Cycles. For conserved binary mixture of spins the ratio of spin up and spin down is taken to be fixed throughout the run as $1: 1$ and in place of spin flip-flop spin interchange move is taken as the trial move. Again the move is accepted if $\exp \left(-\Delta E / k_{B}\right) T \geq p$ in case of conserved spin case otherwise the spins are restored to their original positions. In this case of conserved spins case only weak external magnetic field as $B=0.50 J_{0} / \mu_{B}$ is applied at $k_{B} T=1.0 J_{0}$ in order to study the spin organization and restructuring of spins with aprori assumption that the weak filed has 
not sufficient strength to flip-flop the spins and hence forth the spins remain conserved for all practical purposes. This is likely to exist in the real world.

\section{Results and discussion}

Figures 1(a) \& (b) show the magnetization vs applied external fields at different temperatures and spin-spin coupling constatants respectively for the ferromagnetic case. Fig. 1(c) $\&$ (d) show the magnetization vs applied external fields at different temperatures and spin-spin coupling constants respectively for the anti-ferromagnetic case. These magnetization vs external magnetic filed curves are plotted with $|\varepsilon(A A)|=|\varepsilon(B B)|=|\varepsilon(A B)|$ following the standard Ising model. The curves are in qualitative agreement with theory [6,7]. Fig. 2(a) shows avg.
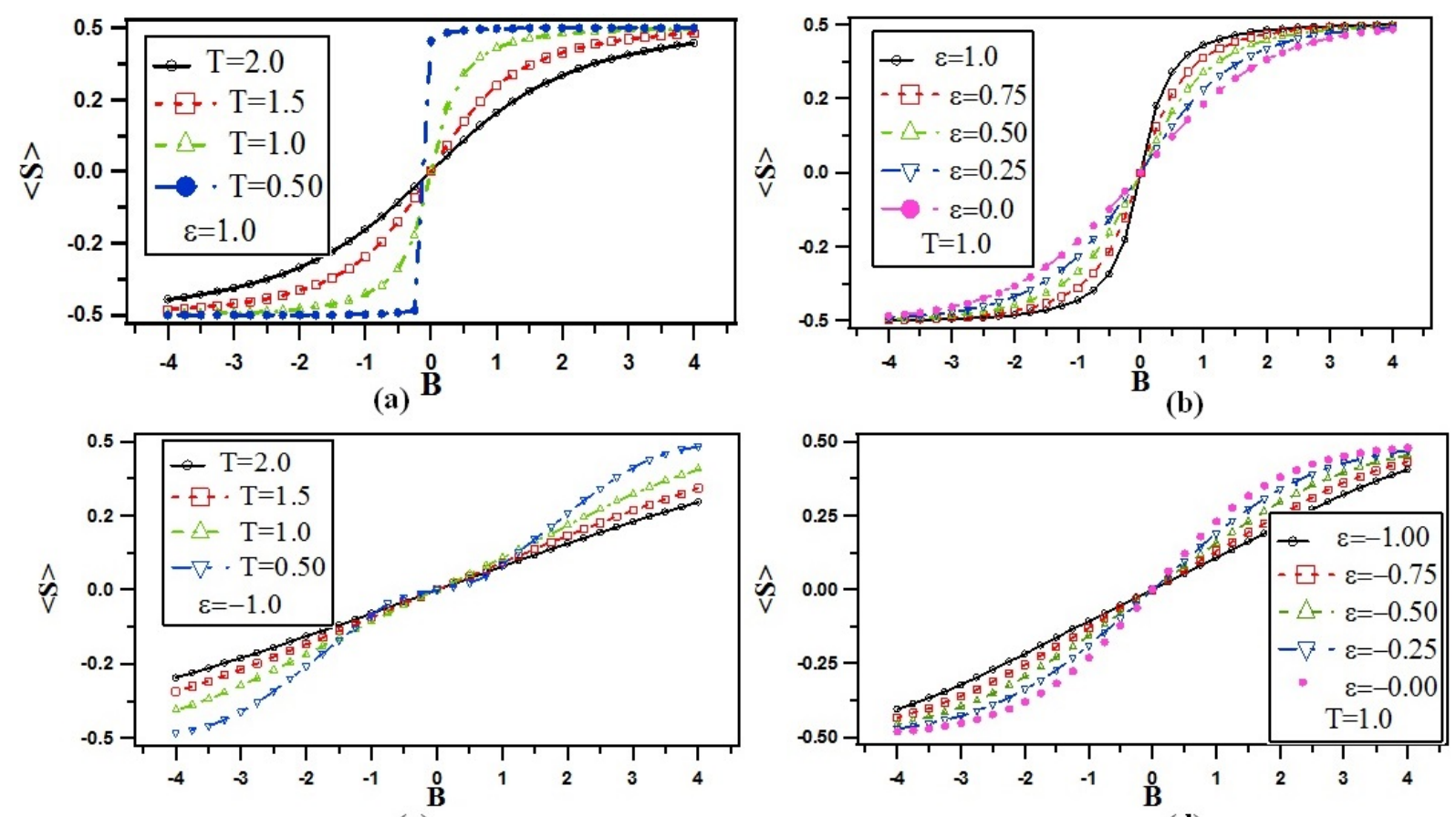

(c)

(d)

FIG. 1. shows the variation of external field vs magnetization a) for different temperatures as $k T=0.50 J_{0}, 1.0 J_{0}, 1.5 J_{0} \& 2.0 J_{0}$ for $\varepsilon(A A)=\varepsilon(B B)=$ $\varepsilon(A B)=\varepsilon=1.0 J_{0}$ for ferromagnetic case; b) for different spin interactions as $\varepsilon(A A)=\varepsilon(B B)=\varepsilon(A B)=\varepsilon=0.25 J_{0}, 0.50 J_{0}, 0.75 J_{0} \& 1.0 J_{0}$ for ferromagnetic case; c) for different temperatures as $k T=0.50 J_{0}, 1.0 J_{0}, 1.5 J_{0} \&$ $2.0 J_{0}$ for $\varepsilon(A A)=\varepsilon(B B)=\varepsilon(A B)=\varepsilon=-1.0 J_{0}$ for anti-ferromagnetic case; d) for different spin interactions as $\varepsilon(A A)=\varepsilon(B B)=\varepsilon(A B)=\varepsilon=-0.25 J_{0}$, $-0.50 J_{0},-0.75 J_{0} \&-1.0 J_{0}$ for anti-ferromagnetic case. $J_{0}=1.0$ is taken to be unity in all cases of study. All the figures shown here for non-conserved spin cases using spin flip-flop method sampled after $20 \mathrm{~K} \mathrm{MC} \mathrm{Cycles}$

energy vs MC Cycles run for the ferromagnteic case. Lowering the temperature results in better spin organization, which in turn, yield lower energies for the system. The effect of temperature on induced magnetization can be observed in Fig. 2(b). This also shows that variations in the repulsive interaction strength between two types of spins i.e. spin up (e.g. type A) and spin down (e.g. type B) also influence the magnetization. Reduced $\varepsilon(A B)$ values enhances the mixing of types A and B spins. The ease of spin mixing lowers the induced magntization. Fig. 2(c) plotted between entropy and MC Cyles looks to support the existence of a super-paramagetic 
type behavior for $\varepsilon(A B)=1.0 J_{0}$ at $k T=2.0 J_{0}$. This indicates rather a surprising trend be-

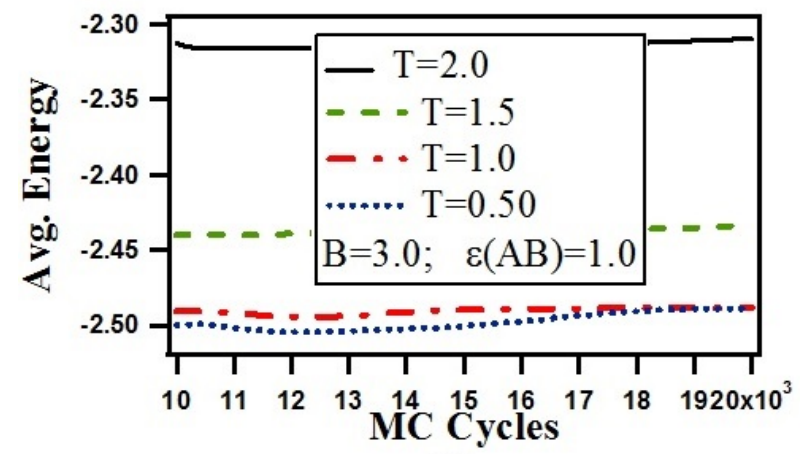

(a)

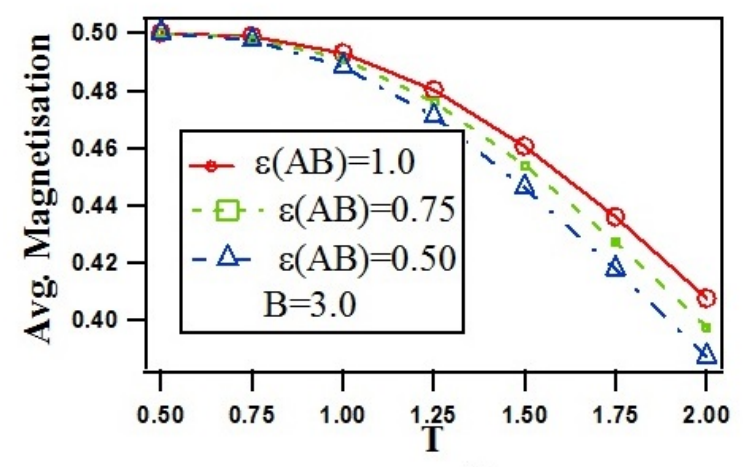

(b)

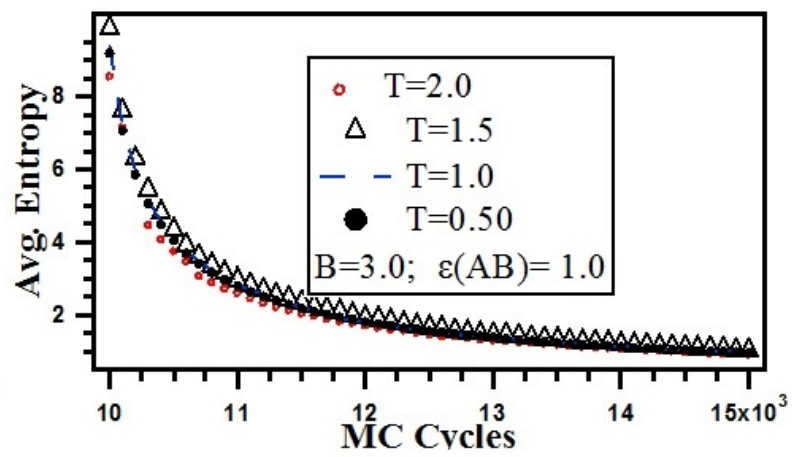

(d)

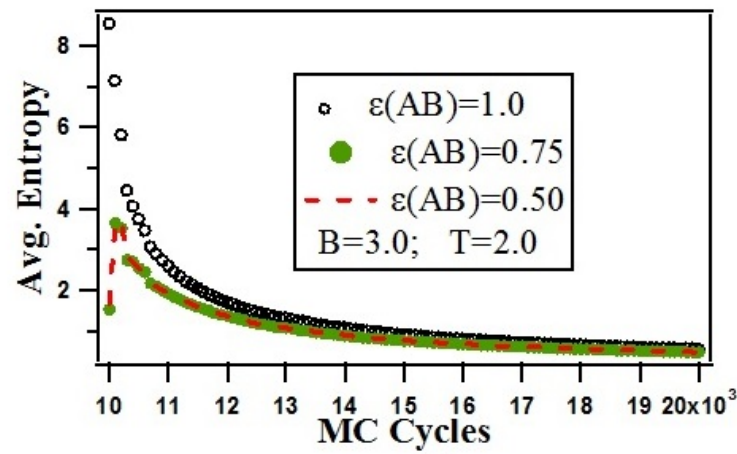

(e)

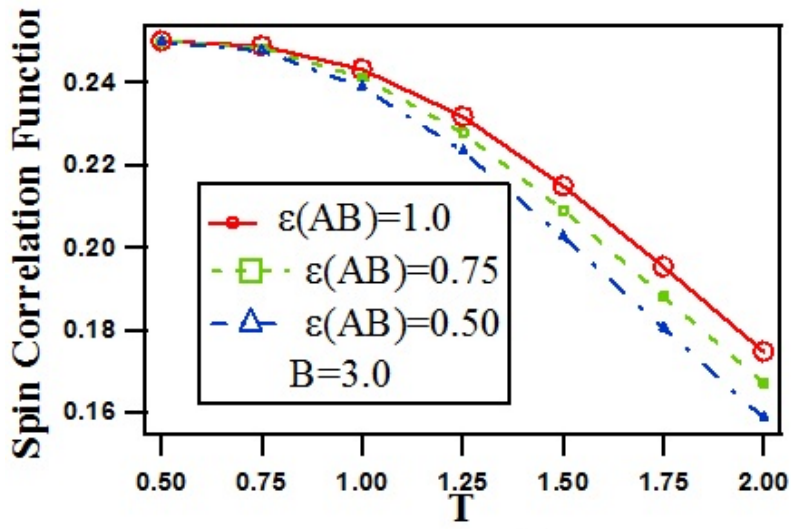

(c)

FIG. 2. Shows the (a) Avg. energy variation with temperature; (b) Variation of induced magnetization vs temperature for different spin interactions $\varepsilon_{A B}=1.0 J_{0}$, $0.75 J_{0} \& 0.50 J_{0}$; (c) Spin correlation function vs temperature for different spin interactions $\varepsilon_{A B}=1.0 J_{0}, 0.75 J_{0}$; (d) Avg. entropy vs temperature and (e) Avg. entropy vs spin interactions $\varepsilon_{A B}$ at $k T=2.0 J_{0}$ for different run cycles. In all cases, the applied external magnetic field is taken as $B=3.0 J_{0} / \mu_{B}$. The data are produced after sampling for $20 \mathrm{~K}$ cycles starting from $10 \mathrm{~K}$ cycles at an interval of 100 cycles. All cases studied here are for non-conserved ferromagnetic film

cause the avg entropy at higher $k T=2.0 J_{0}$ is observed to be lower than that of $k T=1.50 J_{0}$, $1.0 J_{0} \& 0.50 J_{0}$. In general, the entropy or spin disorder must increase at higher temperatures. The lower entropy at relatively higher $k T=2.0 J_{0}$ can be explained on the basis of a super paramagnetic behavior forthe film in which the spin-spin correlation gets weakened to the extent 
that the spin starts following the applied external magnetic field irrespective of the interactions with neighboring spins. For relatively easy mixing of spin up i.e. type A and spin down i.e. type $\mathrm{B}$, the spin correlations seems to increase, resulting in a better organization of spins, thus lowering the entropy of the systems as shown in Fig. 2(d). For $\varepsilon(A B)=0.75 J_{0} \& 0.50 J_{0}$, the entropy first increases for first $1000 \mathrm{MC}$ Cycles and then decreases with subsequent cyles. The relatively very high values of avg. entropy for $\varepsilon(A B)=1.0 J_{0}$ at $k T=2.0 J_{0}$ also supports that at this temperature the film's behavior becomes super paramagnetic. Here, $J_{0} / k$ is chosen to be unity in all the experiments; i.e. $J_{0}=1.0$. Here, the average energy, temperature and external magnetic field are expressed in units of $J_{0}, J_{0} / k_{B}$ and $J_{0} / \mu_{B}$ respectively but are taken to be unity numerically in all the figures of the paper but not shown. Interested readers may determine the exact numerical values provided that they appropriately chose the units of $J_{0}$, $k_{B}$ and $\mu B$ which are taken to be unity in all the cases of study here. This paper is intended to be a qualitative and phenomenological study of the subject and henceforth omitted from the discussion.

Similar avg. energy sampling has been done at $k T=1.0 J_{0}$ for $\varepsilon(A B)=1.0 J_{0}, 0.75 J_{0}$ $\& 0.50 J_{0}$. The reative ease of mixing the two types of spins weaken the ferroic behavior of spins, resulting in slightly higher avg. energy values, as shown in Fig. 3(a). For all the three cases of $\varepsilon(A B)=1.0 J_{0}, 0.75 J_{0} \& 0.50 J_{0}$ at $k T=1.0 J_{0}$ a negative slope is observed in the respective avg. energy values for first few thousand odd MC Cycles which indicate the presence of a minima. The variations aret at the third decimal place and are insignificant for indication of any new physical process.

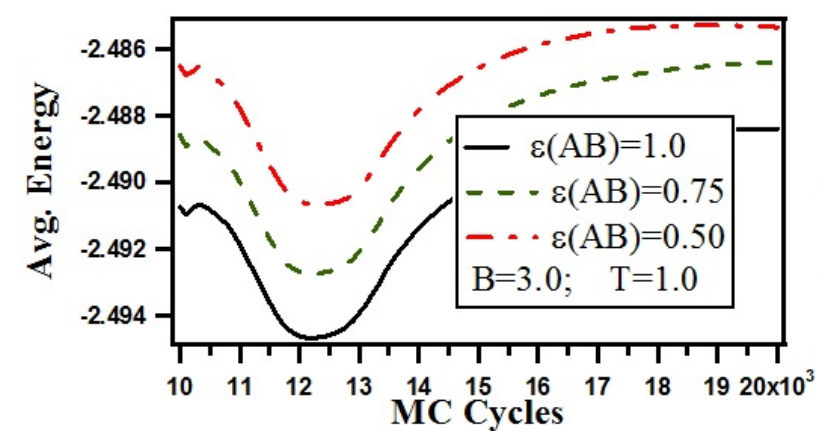

(a)

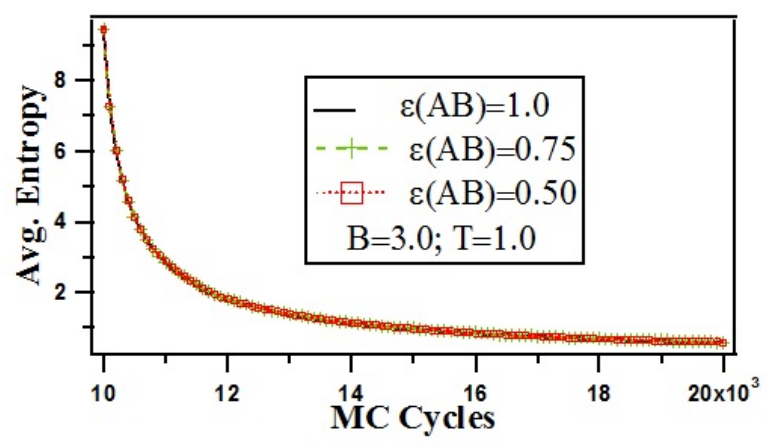

(b)

Fig. 3. Shows (a) Avg. free energy vs spin interactions at different run cycles; (b) Avg. entropy vs spin interactions at different run cycles at $B=3.0 J_{0} / \mu_{B}$ and $k T=1.0 J_{0}$ for non-conserved ferromagnetic case. All data are produced after sampling for $20 \mathrm{~K}$ cycles starting from $10 \mathrm{~K}$ cycles at an interval of 100 cycles. The lines are to aid visualization

The corresponding avg., entropies are plotted in Fig. 3(b). Initially, decreasing energy values were observed for first $10000-12000 \mathrm{MC}$ Cycles for $T=1.0$, as shown in Fig. 3(a). Though the corresponding avg. entropy (depicted in Fig. 3(b)) shows more or less the same values for all three studied cases: $\varepsilon(A B)=1.0 J_{0}, 0.75 J_{0} \& 0.50 J_{0}$ for the same number of cycles run, but the change in entropy $(-\mathrm{ve})$ (i.e. ordering of spins decreases entropy) increases the Helmholtz free energy $\Delta F=\Delta U-T \Delta S$ for a constant internal energy. This can be accounted for on the basis of an energy increase because of homogeneous mixing of up and down spins [7]. Phase separation occurs at relatively lower temperature, which is energetically more favorable and shows a minima in free energy. The remarkable thing to note here is that all the plots of avg. entropy yields entropy values approaching 0 , which indicates that after a 
long run of several cycles, equilibrium is achieved and the existing microstates have probability close to 1.0. The free energy vs MC Cycles and entropy vs MC Cycles for anti-ferromagnetic cases are as shown in Fig. 4(a) and Fig. 4(b) respectively. The effect of temperature on spin correlation of opposite spins in this case is similar to that of the same type of spins in the ferromagnetic case. The average magnetization and spin correlation function are plotted against temperature in Fig. 5(a) and Fig. 5(b) respectively. These are found to be in agreement with qualitative intuition discussed in this paper.

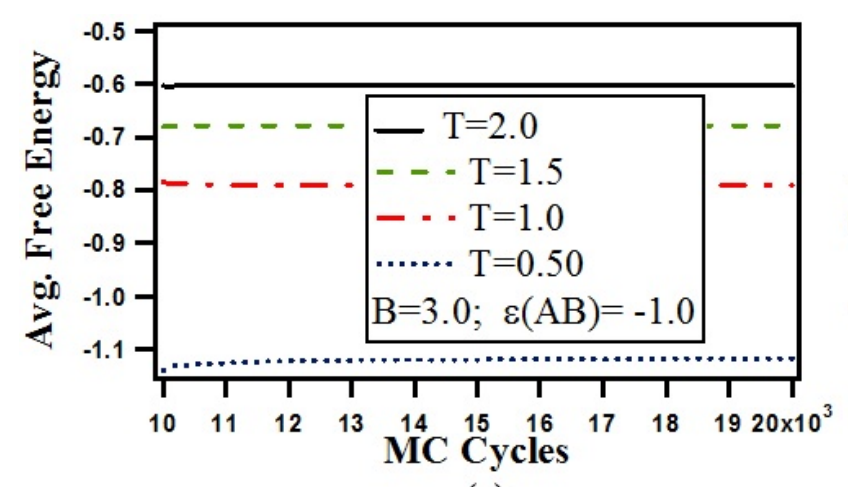

(a)

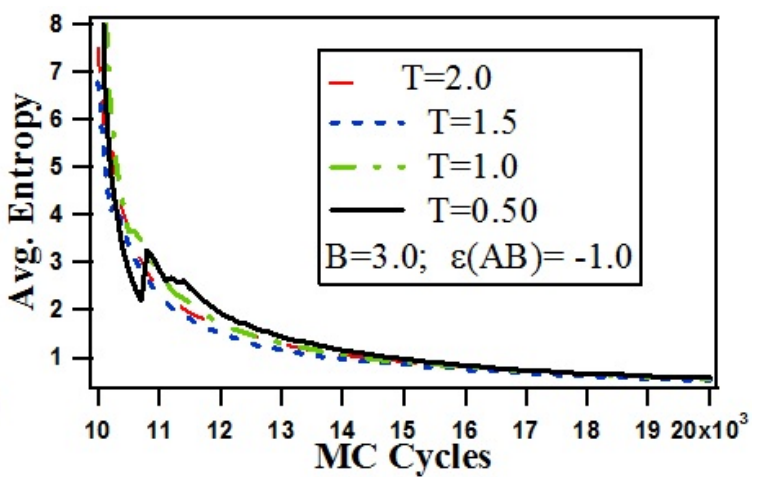

(b)

FIG. 4. Shows (a) Avg. free energy vs temperature at different run cycles; (b) Avg. entropy vs temperature at different run cycles at $B=3.0 J_{0} / \mu_{B}$ for non-conserved anti-ferromagnetic case. All data are produced after sampling for $20 \mathrm{~K}$ cycles starting from $10 \mathrm{~K}$ cycles at an interval of 100 cycles. The lines are to assist in visualization

The ferromagnetic transition temperatures for $\varepsilon(A B)=1.0 J_{0}, 0.75 J_{0} \& 0.50 J_{0}$ are respectively noted as $k_{B} T=0.44 J_{0}, 0.39 J_{0} \& 0.33 J_{0}$ respectively. The spin correlation function diverges at the transition temperatures. The lines are to aid visualization.

The conserved spin case is shown for ferromagnetic case with spin up and spin down ratio $1: 1$ in Figs. 6(a), 6(b), 6(c) and 6(d). The conserved spin case for anti-ferromagnetic case with a $1: 1$ spin up and spin down ratio is shown in Figs. 6(a) \& 6(b). The large drop at lower temperature near $k T=0.50 J_{0}$ in average energy values indicates a spontaneous ordering in both cases (dominantly in case of ferromagnetism). The spin-spin correlation first increases at lower temperatures and then falls after definite temperatures (i.e. the temperature where the spontaneous ordering switches off Fig. 8(a). The two regimes shows two distinct and opposite behaviors. For the anti-ferromagnetic case, the converse is true but interestingly for $\varepsilon(A B)=-0.50 J_{0}$ with $\varepsilon(A A)=\varepsilon(B B)=-1.0 J_{0}$ the spin-spin correlation alters the trend to match with that of the ferromagnetic case. Fig. 6(c) shows a change in the trend of average energy values for conserved ferromagnetic case for $\varepsilon(A B)=1.0 J_{0} \& \varepsilon(A B)=0.75 J_{0}$ at weak external magnetic field of $B=0.50 J_{0} / \mu_{B}$ in films [18].

Even though the spin-spin correlation for like and opposite spins is high (i.e. $\varepsilon(A B)=1.0 J_{0}$, that should enhance the coarsening of similar spins), the average energy values obtained are low (-ve values) in comparison to that of $\varepsilon(A B)=0.75 J_{0}$ which allows for relatively easier coarsening of similar spins). This type of change can be explained on the basis of domain stress which depends on the domain energy. The formation of domains in presence of an external magnetic field deforms the film, creating an anisotropy directed by the external magnetic field. The magnetoelastic energy seems to play an important role. Z. Malek and W. Schiipel et al. have proposed a hypothesis regarding the uni-axial anisotropic effect about the easy axis (i.e. perpendicular to the direction of the applied magnetic field) produced in 


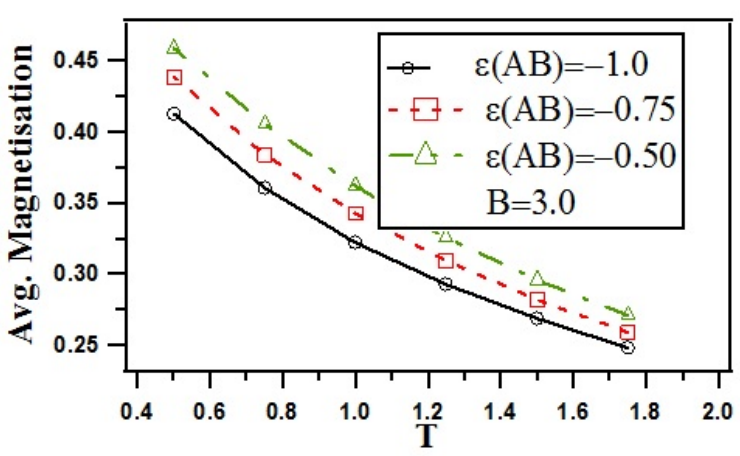

(a)

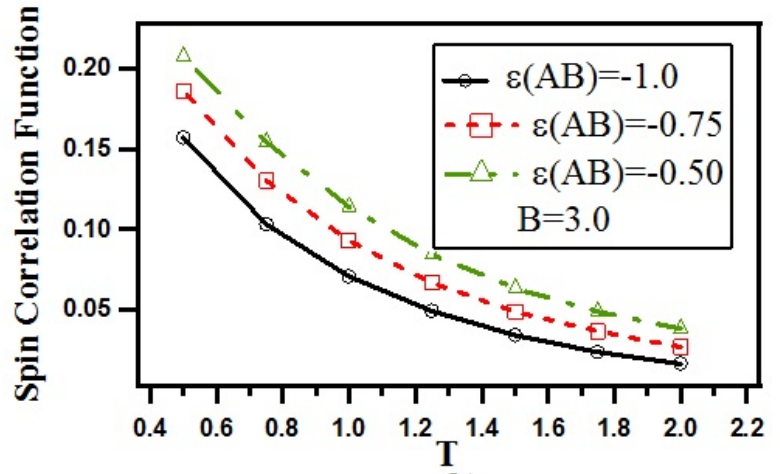

(b)

FIG. 5. Shows (a) Avg. magnetization vs temperature for different spin interactions \& (b) Spin correlation function vs temperature for different spin interactions at $B=3.0 J_{0} / \mu_{B}$. All data are produced after sampling for $20 \mathrm{~K}$ cycles starting from $10 \mathrm{~K}$ cycles at an interval of 100 cycles. All cases studied here are for non-conserved anti-ferromagnetic film. The lines are to aid visualization

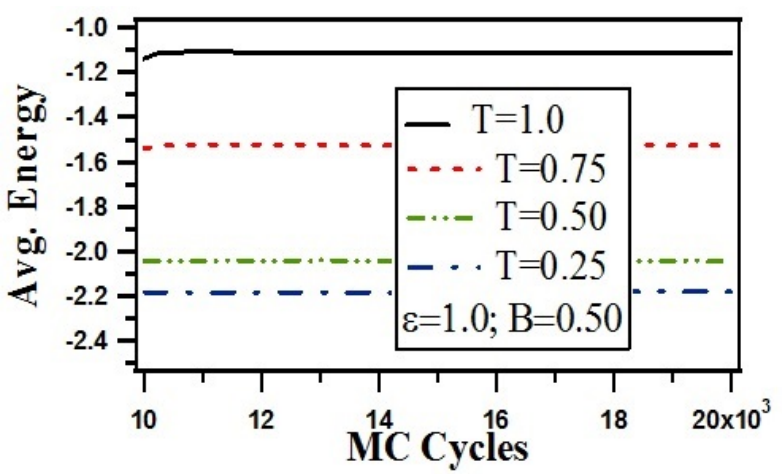

(a)

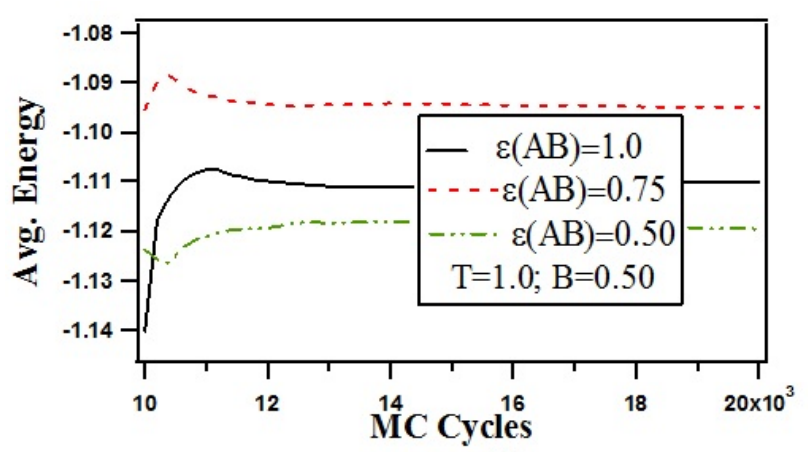

(c)

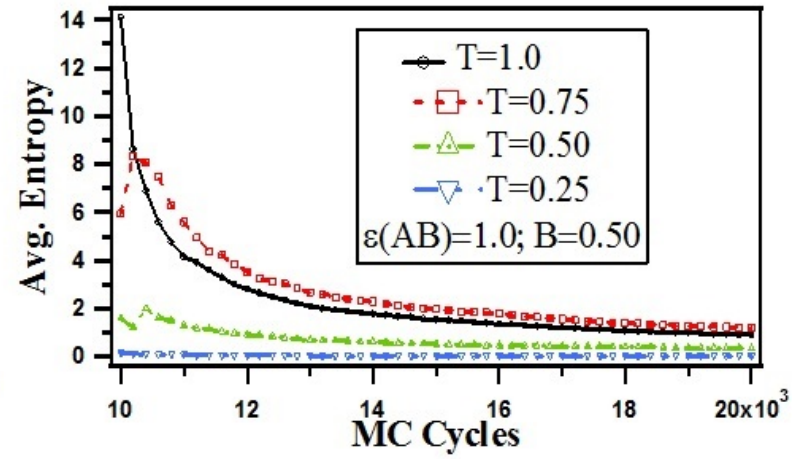

(b)

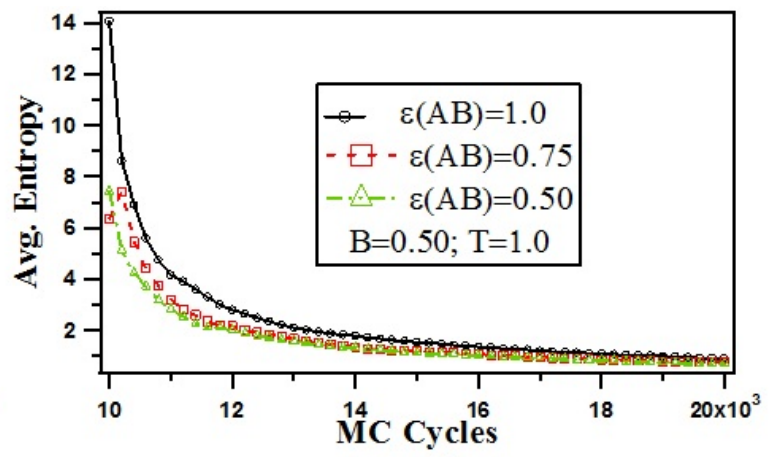

(d)

Fig. 6. Shows (a) Avg. energy vs MC Cycles at different $T$; (b) Avg. energy vs MC Cycles at different $\varepsilon(A B)$; (c) Avg. entropy vs MC Cycles at different $T$; (d) Avg. entropy vs MC Cyles at different $\varepsilon(A B)$ for different run cycles at a relatively weak magnetic field $B=0.50 J_{0} / \mu_{B}$ for conserved binary mixture with composition $A: B:: 50: 50$. All data are produced after sampling for $20 \mathrm{~K}$ cycles starting from $10 \mathrm{~K}$ cycles at an interval of 100 cycles. All cases studied here are for conserved spin ferromagnetic film. The lines are to aid visualization 


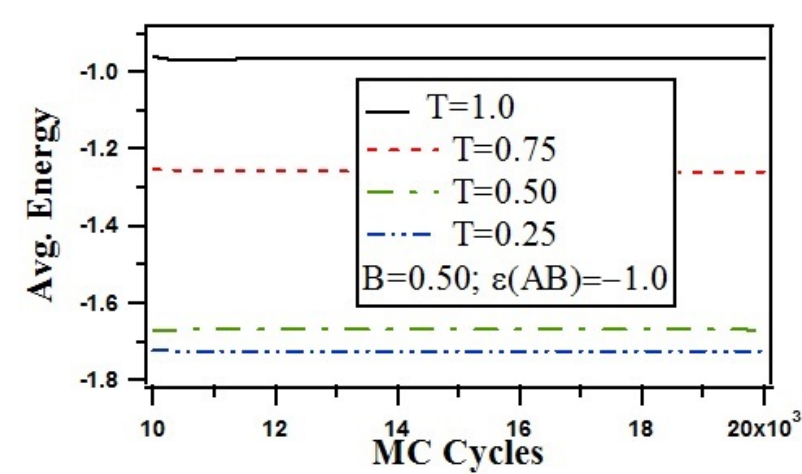

(a)

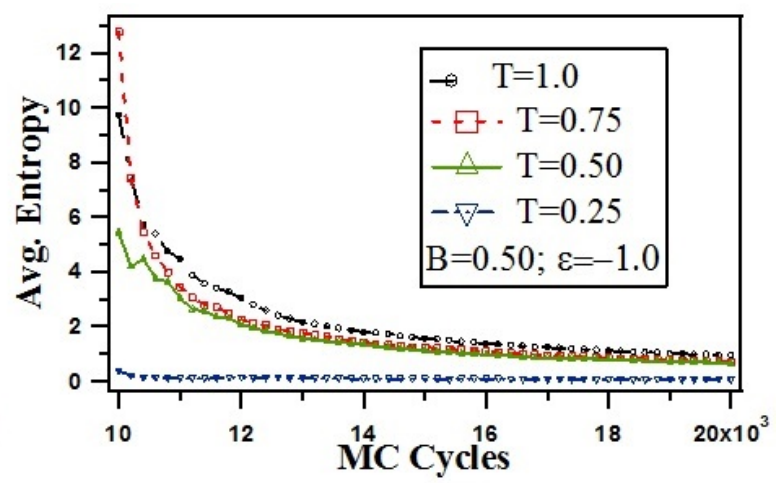

(b)

FIG. 7. Shows (a) Avg. energy vs temperature \& (b) Spin correlation function vs temperature for different run cycles at a relatively weak magnetic field $B=0.50 J_{0} / \mu_{B}$ for conserved binary mixture with composition $A: B:: 50: 50$. All data are produced after sampling for $20 \mathrm{~K}$ cycles starting from $10 \mathrm{~K}$ cycles at an interval of 100 cycles. All cases studied here are for conserved antiferromagnetic film. The lines are to aid visualization

ferromagnetic thin films [18]. Only weak field is taken in closer proximity to real systems. Constraint of spin conservation cannot be really permissible with higher strength of magnetic fields.

The lowest energy magnetization texture depends on the shape of the domain formed [19]. At relatively lower temperatures, the spin ordering attains equilibrium quickly within $10 \mathrm{~K} \mathrm{MC}$ cycles and large changes in entropy are not observed with run cycles because of the reduced mobility for the spins, which is temperature-dependent. The simulation data below $k T=0.30 J_{0}$ may not be reliable, because of the apparent presence of freezing type of behavior, which means that quantum mechanical treatment may be required in order to draw any meaningful conclusions. Fig. 7 shows (a) avg. energy vs temperature and (b) spin correlation function vs temperature for different run cycles at a relatively weak magnetic field $B=0.50 J_{0} / \mu B$ for conserved binary mixture with composition $A: B:: 50: 50$. All data are produced after sampling for $20 \mathrm{~K}$ cycles starting from $10 \mathrm{~K}$ cycles at an interval of 100 cycles. All cases studied here are for conserved anti-ferromagnetic film. These curves support the effect of temperature on average energy and entropy of the system as discussed in the earlier cases of studies. Fig. 8(a) and Fig. 8(b) respectively shows the spin correlation functions for conserved ferromagnetic an conserved anti-ferromagnetic cases respectively. The ferromagnetic case shows a maximum near the spontaneous transition temperatures and decreases when away from it. This is in agreement with theory. The anti-ferromagnetic case shows a negative effect. The figures show interesting interplay of exchange interactions depending on temperature.

The spontaneous magnetization is observed in the absence of an external magnetic field i.e. $B=0.0 J_{0} / \mu_{B}$ for non-conserved binary mixture ferromagnetic film as shown in Fig. 9 and Fig. 10. All data are produced after sampling for $20 \mathrm{~K}$ cycles starting from $10 \mathrm{~K}$ cycles at an interval of 100 cycles. The spontaneous ferromagnetic transition temperatures for $\varepsilon(A B)=1.0 J_{0}, 0.75 J_{0} \& 0.50 J_{0}$ are respectively noted as $k_{B} T=0.44,0.39$ and 0.33 . The spin correlation function diverges at these transition temperatures, which can be understood as theoretical evidence in support of the spontaneous magnetization observation. The micrographs for conserved ferro- and anti-ferromagnetic films are shown in Figs. 11(a) and 11(b) respectively. The micrographs for non-conserved ferro- and anti-ferromagnetic films are shown in Figs. 12(a) 


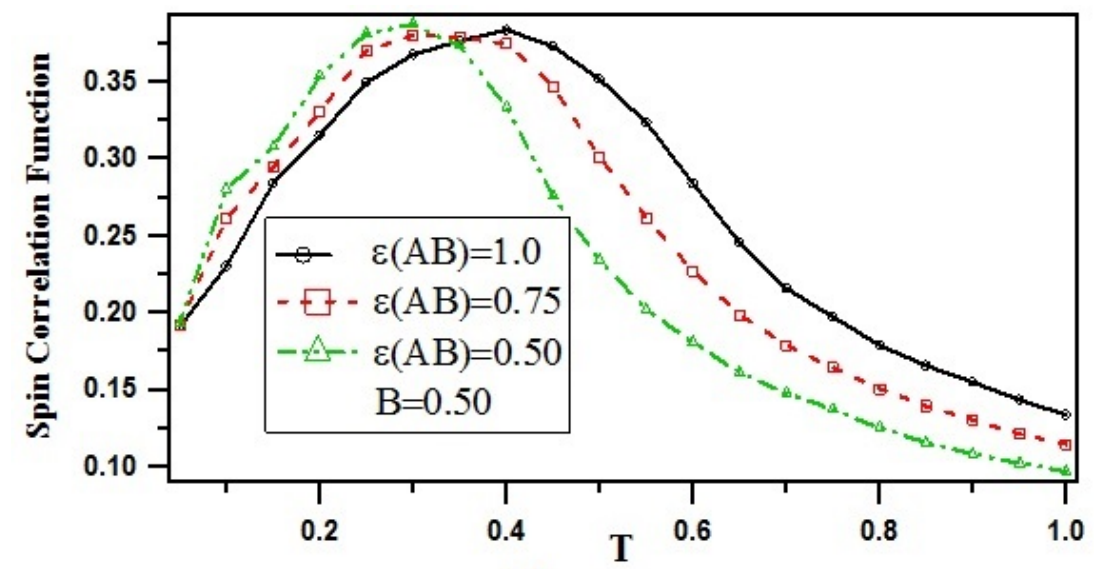

(a)

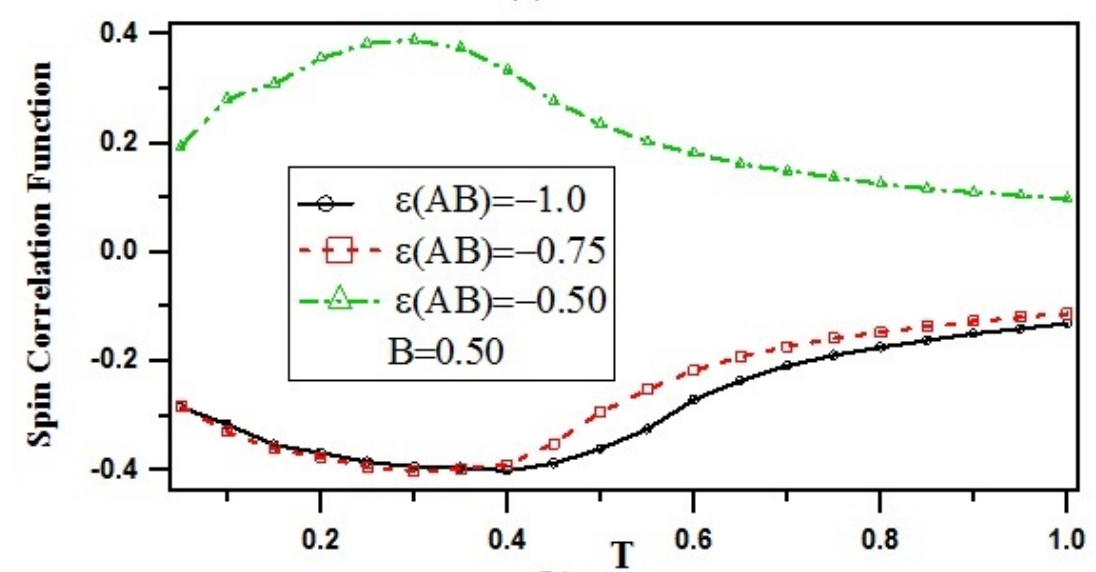

(b)

FIG. 8. Shows (a) Spin correlation function vs $T$ for different $\varepsilon(A B)=1.0 J_{0}$, $0.75 J_{0} \& 0.50 J_{0}$ for conserved ferromagnetic film; (b) Spin correlation function vs $T$ for different $\varepsilon(A B)=-1.0 J_{0},-0.75 J_{0} \&-0.50 J_{0}$ for conserved antiferromagnetic film at a relatively weak magnetic field $B=0.50 J_{0} / \mu_{B}$. All data are produced after sampling for $20 \mathrm{~K}$ cycles starting from $10 \mathrm{~K}$ cycles at an interval of 100 cycles. The lines are to aid visualization

and 12(b) respectively. Fig. 12(b) clearly indicates a well-defined domain formation, hinting at the presence of an anisotropic effect, even at an external magnetic field $B=0.0 J_{0} / \mu_{B}$ because of the fact that the system is kept below the spontaneous transition temperature. The ferromagnetic to paramagnetic transitions are not very sharp, but the range of the spin-spin interaction can be said to decay gradually. Even at higher temperatures, as $k T=2.0 J_{0}$, the opposite spin pair correlation function Fig. 14 supports the formation of tiny domains with spin transport from one domain to another whereas for lower temperatures, below $k T=1.0 J_{0}$, the presence of a majority of $+1 / 2$ spins diminishes the effect. Tiny domain walls have lower energy surrounded by opposite spins and seem to be energetically preferred. This quasi nature of spin-spin interaction with temperature is also supported by the corresponding ensemble average of entropies. The simulation for the ensemble average of entropy is repeated with $50 \mathrm{~K} \mathrm{MC}$ Cycles in which the data is sampled after every $100 \mathrm{MC}$ Cycles starting from $30 \mathrm{~K} \mathrm{MC} \mathrm{Cycles}$ in order to ensure that no aging effects are present. This is shown in Fig. 13. These results were found to be in very good quantitative agreement with Fig. 2(d) with only $20 \mathrm{~K} \mathrm{MC}$ Cycles. Fig. 15 shows the magnetic susceptibility vs temperature. Doing so helps to study the underlying 
physical phenomena in three cases of magnetization i.e. ferromagnetic, anti-ferromagnetic and paramagnetic. Spontaneous magnetization is observed below a critical temperature $T_{c}$ known as the Curie temperature. The ferromagnetic material becomes paramagnetic above the Curie temperature. In the case of anti-ferromagnetic material, the magnetic susceptibility $\chi$ initially increases with temperature and after attaining a critical temperature, $\chi$ decreases Fig. 15. The critical temperature is called the Neel temperature. Spin up and spin down pairs try to remain intact at lower temperatures. The magnetic induction is strong but negative. Hence the magnetization in external field is small at lower temperatures. With increase in temperature, the spin-spin interaction becomes small and hence $\chi$ increases [20]. Above a certain high temperature, known as the Neel temperature, the spins gradually become free and the material becomes paramagnetic and $\chi$ decreases with temperature.

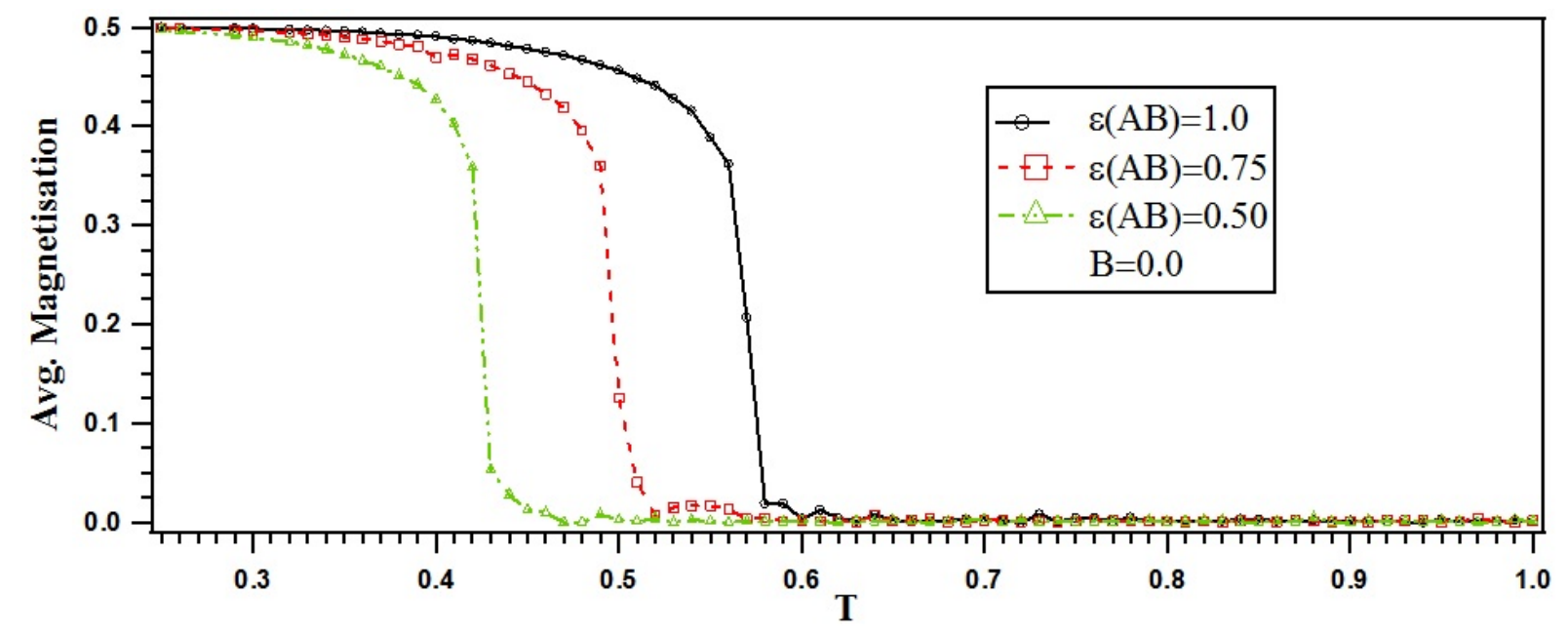

FIG. 9. Shows the spontaneous magnetization in the absence of an external magnetic field i.e. $B=0.0 J_{0} / \mu_{B}$ for non-conserved binary mixture with initial composition $A: B$ :: 50 : 50 i.e. random binary mixture. All data are produced after sampling for $20 \mathrm{~K}$ cycles starting from $10 \mathrm{~K}$ cycles at an interval of 100 cycles. All cases studied here are for non-conserved ferromagnetic film. The spontaneous ferromagnetic transition temperatures for $\varepsilon(A B)=1.0 J_{0}, 0.75 J_{0}$ $\& 0.50 J_{0}$ are observed as $k_{B} T=0.44 J_{0}, 0.39 J_{0} \& 0.33 J_{0}$ (i.e. nearly $96 \%$ magnetizations are observed at these temperatures) respectively at $B=0.0 J_{0} / \mu_{B}$ (i.e. absence of any external magnetic field). The lines are to aid visualization

\section{Conclusion}

This paper deals with the numerical results for the simulation of magnetization in $2 \mathrm{D}$ thin films, highlighting the competitiveness of ensemble free energy and entropy averages. Though, many experimental research papers [21-23] have been published showing the magnetic properties of the binary mixtures, discussions of their thermodynamic properties have been scarce. The simulation techniques are also not used in published and available reports to give insight into this type of interplay between free energy and entropy, especially for spin mixture in the absence or presence of an external field. Though the author observes some finite size effect but it has been found that increasing size to $200 \times 200$ does not yield much change. The fluctuations are high at $T=2.0$, indicating a super paramagnetic type of behavior in our case. The spin fluctuations in $2 \mathrm{D}$ thin films may give rise to significant variations 


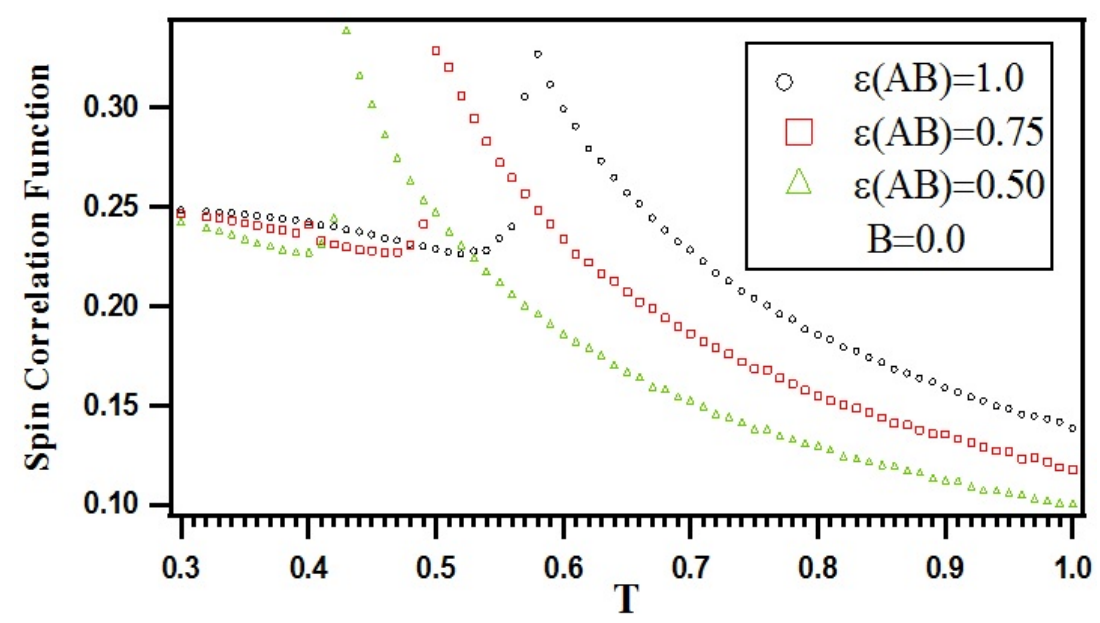

FIG. 10. Confirms the spontaneous magnetization in absence of external magnetic field i.e. $B=0.0 J_{0} / \mu_{B}$ for non-conserved binary mixture with initial composition $A: B$ :: 50 : 50 i.e. random binary mixture. All data are produced after sampling for $20 \mathrm{~K}$ cycles starting from $10 \mathrm{~K}$ cycles at an interval of 100 cycles. All cases studied here are for ferromagnetic film

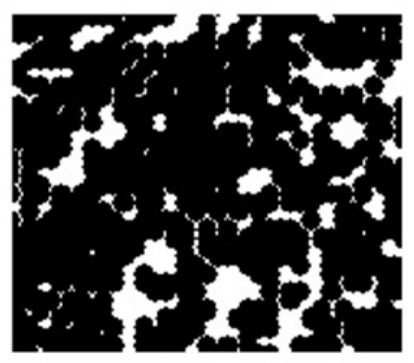

(a)

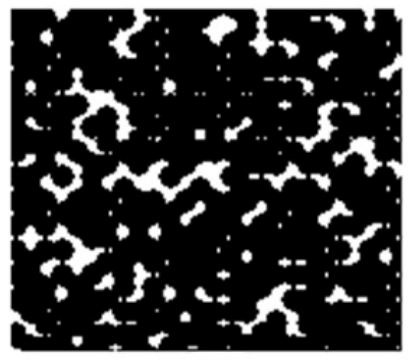

(b)

FIG. 11. Shows the (a) micrograph at $k T=1.0 J_{0}$ for conserved ferromagnetic case and (b) micrograph at $k T=1.0 J_{0}$ for conserved anti-ferromagnetic case. The configuration data are produced after sampling for $20 \mathrm{~K}$ cycles. For ferromagnetic case large circular patches of spin-down component are observed whereas for anti-ferromagnetic case small circular patches of spin-down component are observed with presence of long ridges in the micrograph which is an indication of wetting phenomena as the ridge interface can host spin-up component which is preferred in the anti-ferromagnetic case. Smaller circular drops in large number can better help the system to achieve equilibrium with minimum possible energy, which is opposite to that of the ferromagnetic case

in the magnetic and electrical properties of 2D thin films which may have a wide range of applications. The ferromagnetic to paramagnetic transitions are not very sharp, but the range of the spin-spin interaction can be said to decay gradually. Even at higher temperatures, as $k T=2.0 J_{0}$, the opposite spin pair correlation function supports the formation of tiny domains with spin transport from one domain to another, whereas for lower temperatures, i.e. below $k T=1.0 J_{0}$, the presence of majority $+1 / 2$ spins diminishes the effect. Tiny domain walls have lower energy surrounded by opposite spins and seem to be energetically preferred. This quasi nature of spin-spin interaction with temperature is also supported by the corresponding ensemble average of entropies. 


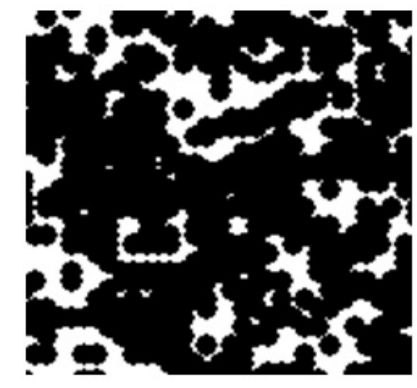

(a)

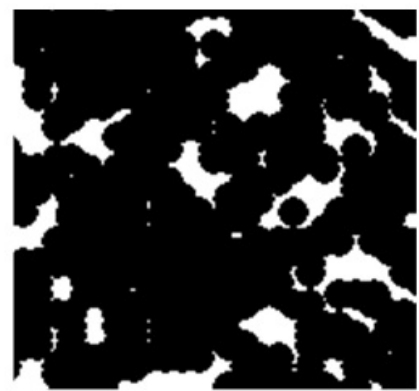

(b)

FIG. 12. Shows the (a) micrograph at $k T=1.0 J_{0}$ for non-conserved ferromagnetic case and (b)!micrograph at $k T=0.45 J_{0}$ for non-conserved ferromagnetic case i.e. below spontaneous transition temperature. The configuration data are produced after sampling for $20 \mathrm{~K}$ cycles. The agitated magnetic domains can be observed at $k T=1.0 J_{0}$ in form of long ridges. At $k T=0.45 J_{0}$, the well-formed domain reflects spin coarsening, which evolves to form nearly circular domains
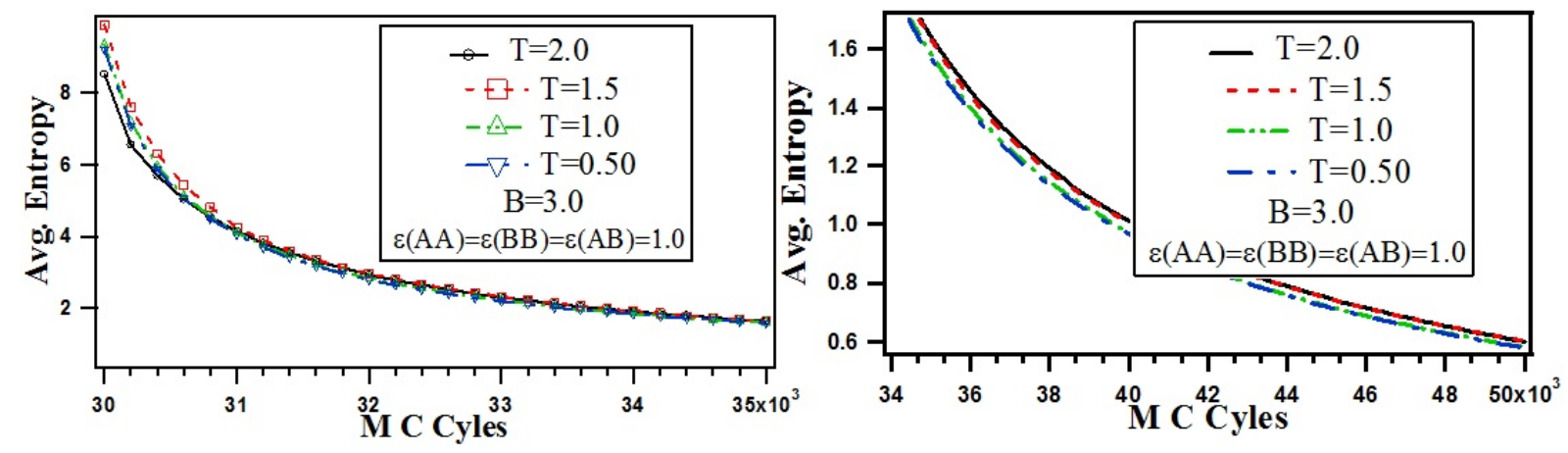

FIG. 13. Shows the avg. entropy vs temperature for the non-conserved ferromagnetic case $\varepsilon_{A B}$ at $k T=2.0 J_{0}$ for different run cycles. In all cases, the applied external magnetic field is taken as $B=3.0 J_{0} / \mu_{B}$. The data are produced after sampling for $20 \mathrm{~K}$ cycles starting from $30 \mathrm{~K}$ cycles at an interval of $100 \mathrm{cy}-$ cles

\section{Acknowledgement}

The author is thankful to University Grant Commission, New Delhi, India for its financial support under UGC, MRP Grant No. 42-849/2013(SR). 

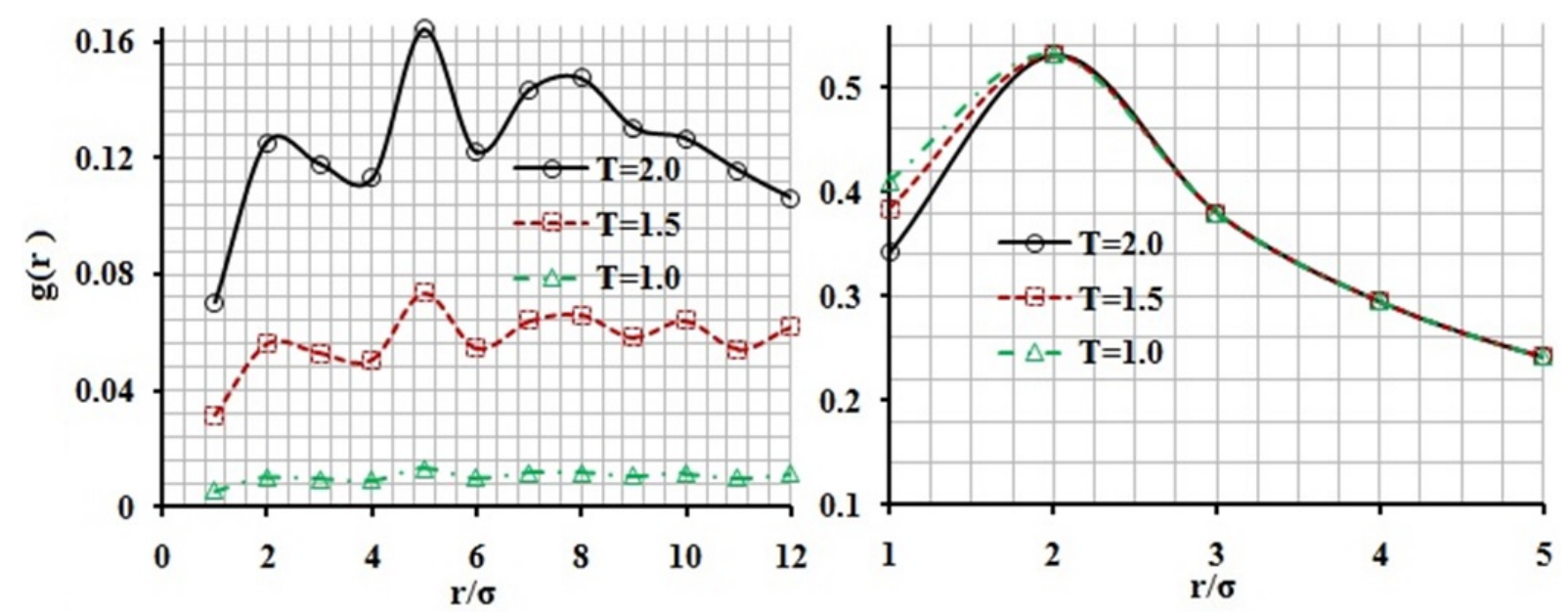

FIG. 14. Shows the pair correlation function $g(r)$ vs distance for opposite spins (left) and for spin up (right) for the non-conserved ferromagnetic case $\varepsilon_{A B}=$ $1.0 J_{0}$ at $k T=1.0 J_{0}, 1.5 J_{0} \& 2.0 J_{0}$. In all cases, the applied external magnetic field is taken as $B=3.0 J_{0} / \mu$. The data are produced after sampling for $20 \mathrm{~K}$ cycles starting from $30 \mathrm{~K}$ cycles at an interval of 100 cycles

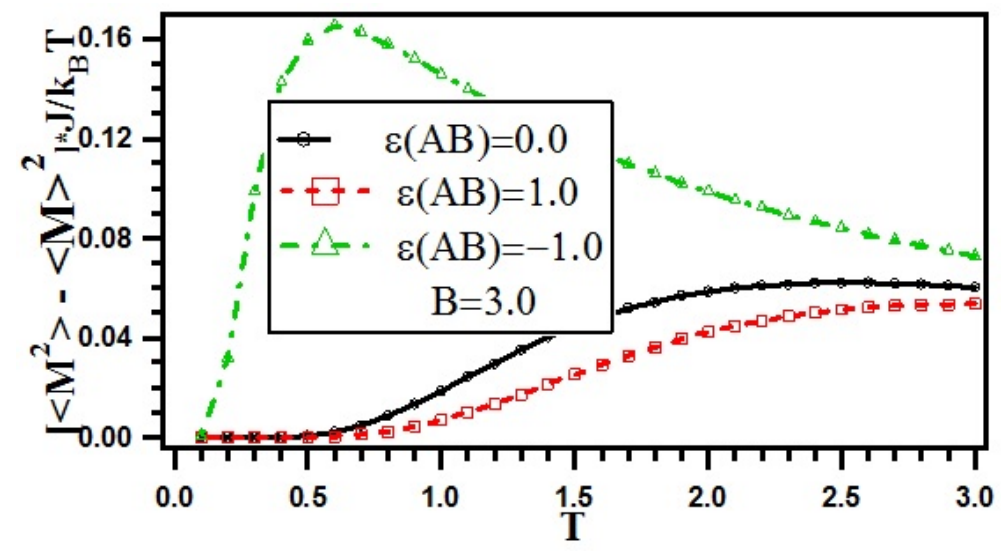

FIG. 15. Shows the magnetic susceptibility vs temperature non-conserved paramagnetic, ferromagnetic \& anti-ferromagnetic case respectively with $\varepsilon_{A B}=0.0 J_{0}$, $1.0 J_{0} \&-1.0 J_{0} ; \varepsilon_{A A}=\varepsilon_{B B}=1.0 J_{0}$. In all case the applied external magnetic field is taken as $B=3.0 J_{0} / \mu$. The data are produced after sampling for $10 \mathrm{~K}$ cycles starting from $10 \mathrm{~K}$ cycles at an interval of 100 cycles

\section{References}

[1] C.P. Pooley, F.J. Owens. Introduction to Nanotechnology, Wiley India Pvt. Ltd. New Delhi, 2009.

[2] J. Ramsden. Nanotechnology: An Introduction, Chapter-2, The nanoscle, Elsevier, UK, 2011.

[3] M. Kuno. Introductory Nanoscience, Garland Science, Taylor and Francis Gropu, USA, 2012.

[4] C.W.Shong, S.C.Haur, A.T.S. Wee. Science at Nanoscale, Pan Stanford Publishing Pvt. Ltd. 2010, Singapore.

[5] R.K. Pathria, P.D. Beale. Statistical Mechanics, 3rd Ed., Butterworth-Heinemenn, USA, 2011.

[6] B.K. Tanner. Introduction to the Physics of Electrons in Solids, Cambridge University Press, UK, 1995.

[7] J.D. Fast. Entropy, 2nd Ed., Gordon and Breach Science Publishers Inc., USA, 1968.

[8] A.K. Saha, S.P. Singh, J.K. Singh, S.K. Kwak. Quasi- 2D and prewetting transitions of square well fluids on a square-well substrate. Molecular Physics, 2009, 107 (20), P. 2189-2200.

[9] S.P. Singh, J.K. Singh, A. Sharma. Adsorption of gas like molecules on self aligned square well fluid channels under confinement of chemically patterned substrates. Appl. Nanosc., 2013, 3, P. 179-187. 
[10] S.P. Singh, J.K. Singh, A. Sharma. Investigating bridge like structures in a square well binary mixture using NVT Monte Carlo simulation. Int. J. Nanosc., 2011, 10, (1), P. 329-333.

[11] S.P. Singh. Spinodal theory: A common rupturing mechanism in spinodal dewetting and surface directed phase separation (some technological aspects and the significance of dipole-quadrupole interaction in spinodal dewetting). Advances in Cond. Mat. Phys., 2011, 2011, Article ID 526397, 14 pages.

[12] S.P. Singh. Spatial correlation in 2D and 3D thin films of conserved binary mixtures in presence of wetting of substrates by preferred majority component: Interpretation in real scenario. Appl. Nanosc., 2012, 2, P. 365369.

[13] S.P. Singh. Revisiting 2D lattice based spin flip-flop Ising model: Magnetic properties of a thin film and its temperature dependence. Eur. J. Phys. Educ., 2014, 5 (3).

[14] S.P. Singh. Dimensions and Trends of Nanotechnology. ISST J. Appl. Phys., 2015, 6 (1), P. $73-77$.

[15] G.H. Wannier. Elements of solid state theory, Chapter-4, Co-operative phenomena in solids. Cambridge University Press, UK, 1960.

[16] H.V. Keer. Principles of solid state, Chapter-5, Magnetic properties. New Age International Publishers, New Delhi, 2005.

[17] J. Marro, R. Toral. Microscopic observations on a kinetic Ising model. Am. J. Phys., 1986, 54 (12), P. 11141121.

[18] V.A. Ignatchenko. Spontaneous magnetization of ferromagnetic thin films. Soviet Physics JETP, 1961, 13 (4), P. 863.

[19] S. Banerjee, M. Widom. Shapes and texture of ferromagnetic liquid droplets. Brazilian J. Phys., 2011,31 (3), P. 360-365.

[20] A. Goswami. Thin film fundamentals, Chapter-12, Magnetic and superconducting film. New Age International Publishers, New Delhi, 2008.

[21] A. Sundaresan, R. Bhargavi, et al. Ferromagnetism as a universal feature of nanoparticles of the otherwise nonmagnetic oxides. Phys. Rev. B, 2006, 74, P. 161306(R).

[22] J.M.D. Coey. High temperature ferromagnetism in dilute magnetic oxides. J. of Appl. Phys., 2005, 97, $10 \mathrm{D} 313$.

[23] N. Poudyai, G.S. Chaubey, C.-B. Rong, J.P. Liu. Shape control of FePt nanocrystals. J. Appl. Phys., 2009, 105, 07A749. 